\title{
The Cholegas Study: safety of prophylactic cholecystectomy during gastrectomy for cancer: preliminary results of a multicentric randomized clinical trial
}

\author{
Marco Bernini - Lapo Bencini • Riccardo Sacchetti - Alberto Marchet · Luigi Cristadoro • Fabio Pacelli • \\ Stefano Berardi - Giovanni B. Doglietto • Fausto Rosa - Giuseppe Verlato • Luca Cozzaglio • Paolo Bechi • \\ Daniele Marrelli • Franco Roviello - Marco Farsi • Italian Research Group for Gastric Cancer (IRGGC)
}

Received: 22 June 2012/ Accepted: 21 August 2012/Published online: 5 September 2012

(C) The International Gastric Cancer Association and The Japanese Gastric Cancer Association 2012

\begin{abstract}
Background Cholelithiasis is more frequent in patients after gastrectomy, due to dissection of vagal branches and gastrointestinal reconstruction.

Methods A randomized controlled trial was conducted from November 2008 to March 2012. Patients were randomized into two groups: prophylactic cholecystectomy (PC) and standard gastric surgery only (SS) for curable cancers. We planned three end points: evaluation of the number of patients who developed symptoms and needed further surgery for cholelithiasis after standard gastric cancer surgery, evaluation of the incidence of cholelithiasis overall after standard gastric cancer surgery and perioperative complications or costs of prophylactic cholecystectomy. The present study answers to the last end point only.
\end{abstract}

Trial Registration: ClinicalTrials.gov ID. NCT00757640.

M. Bernini · L. Bencini $(\varangle) \cdot$ R. Sacchetti · M. Farsi Division of Oncologic Surgery, Department of Oncology, Azienda Ospedaliero, Careggi University Hospital, Largo Brambilla 3, 50134 Florence, Italy

e-mail: lapbenc@tin.it

A. Marchet

Department of Surgery, University Hospital, Padua, Italy

L. Cristadoro

General Surgery, "C. Poma" Hospital, Pieve di Coriano,

Mantua, Italy

F. Pacelli - S. Berardi

Surgical Oncology, Catholic University, Campobasso, Italy

G. B. Doglietto · F. Rosa

Digestive Surgery of University Hospital “A.Gemelli”,

Rome, Italy
Results After 40 months from the beginning of study, 172 patients were eligible from 9 Centers. Ten patients refused consent and 32 were excluded due to flawing of inclusion criteria (not confirmed adenocarcinomas and no R0 surgery). Therefore, final analysis included 130 patients: 65 in PC group and 65 in SS. Among PC group, 12 patients had surgical complications during the perioperative period; only 1 biliary leakage, conservatively treated, might have been caused by prophylactic cholecystectomy. 6 patients had surgical complications in SS group. One postoperative death occurred in PC group due to pulmonary embolism. Differences were not statistically significant. Similarly, no differences were significant in duration of surgery, blood loss, hospital stay.

Conclusions Concomitant cholecystectomy during standard surgery for gastric malignancies seemed to add no extra perioperative morbidity, mortality and costs to the sample included in the study.

\author{
G. Verlato \\ Unit of Epidemiology and Medical Statistics, \\ University of Verona, Verona, Italy \\ L. Cozzaglio \\ Division of Surgical Oncology, Humanitas Hospital, Milan, Italy \\ P. Bechi \\ General Surgery, Careggi University Hospital, Florence, Italy \\ D. Marrelli · F. Roviello \\ Department of Surgery, University of Siena, Siena, Italy
}


Keywords Prophylactic cholecystectomy · Gastric cancer surgery $\cdot$ Randomized trial

\section{Introduction}

The incidence of gallstones and gallbladder sludge has been reported for many years to be higher in patients after gastrectomy than in general population. Many studies reported a three-four fold increased incidence 5 years after gastric surgery (up to 15-25\%) [1-6] with an estimated mean value of $17 \%$ in all studies [1-7]. The underlying mechanism was thought to be related to surgical dissection of the vagus nerve branches and the anatomical gastrointestinal reconstruction [5, 8-10], and to the extent of gastrectomy and lymphadenectomy $[1,4,11-13]$.

Due to such higher rate of gallstones, some Authors proposed prophylactic cholecystectomy, advocating that concomitant cholecystectomy is not time consuming and substantially with minimal additional risks for the patients [14-16], while subsequent cholecystectomy (in most cases attempted laparoscopically) is known to be more challenging after gastric surgery, with an increased risk of conversion, bile duct injuries and a longer operating time $[17,18]$. Another advantage of prophylactic cholecystectomy was represented by the avoidance of a possible reintervention in case of acute post-operative cholecystitis $[7,19,20]$ and the avoidance of difficult procedures like endoscopic retrograde cholangiography (ERC) in the presence of Roux-en-Y reconstruction.

On the other hand, not all the patients who are diagnosed to have cholelithiasis after gastric cancer surgery will develop symptoms or require additional surgical treatments [2-4] and a standard laparoscopic cholecystectomy is feasible even in those patients who underwent previous gastric surgery [17, 21]. Moreover, in recent years, the problem of litigation and malpractice claims have raised the issue of removing a normal organ for prophylaxis of any benign disease. However, new ongoing technologies and chemotherapies have prolonged survival in those operated for gastric malignancy, increasing the possibility of gallstones development after surgery. Although in those patients operated for gastric cancer the 5-year survival rate is less than $50 \%$ in many centers [22, 23], more than $90 \%$ of those who will develop calculi, will do it within 2 years after surgery [4]. A recent, well-conducted review article on simultaneous/incidental cholecystectomy during gastro-esophageal resection concluded that only less than $5 \%$ of those patients operated on required a subsequent cholecystectomy for symptoms of cholelithiasis, mostly laparoscopically and with few additional morbidity and mortality [7].
Due to the lack of specific data and to the presence of contrasting studies, some authors $[4,11]$ recommend to perform a prophylactic cholecystectomy at the time of gastric surgery to avoid complications and impairment of quality of life in surviving patients, while others do not $[1,7]$ and the decision of gallbladder management is left, to date, to each surgeon's preference.

A randomized multicentric controlled trial on this topic began on November 2008. The present study, at the end of recruitment, was aimed to present preliminary results in terms of perioperative complications rate, operative time and postoperative stay.

\section{Methods}

\section{Trial design}

The Cholegas Study was approved by the ethical Committee of each participating center since October 2008. Details of the study protocol was published elsewhere [24]. Patients suitable for curative surgery, and $\leq 80$ years old, with an histopathological confirmed gastric adenocarcinoma, were considered eligible for the study and asked to participate after reading an abstract of the protocol. Those who refused to sign the consent, had a previous cholecystectomy, demonstrated cholelithiasis or biliary sludge, metastases or metabolic diseases that may favor gallstones formation (such as hemolytic anemia and genetic hypercholesterolemia) were excluded.

Originally, the study had three planned end points: (1) evaluation of the number of patients who developed symptoms and needed further surgery for cholelithiasis after standard gastric cancer surgery. (2) Evaluation of the incidence of cholelithiasis overall after standard gastric cancer surgery. (3) Perioperative complications and costs of prophylactic cholecystectomy. The present study answers to the last end point only, while an appropriate follow-up is still necessary for the other end points.

In brief, we assumed that the proportion of patients free from cholelithiasis was $100 \% 5$ years after gastrectomy plus prophylactic cholecystectomy. We did not consider any other complications from common bile duct stones, because there is no literature of such a disease in patients with a normal gallbladder (inclusion criterion) who underwent prophylactic cholecystectomy during gastric cancer surgery. On the other hand, $20 \%$ was the proportion assumed of cholelithiasis in patients undergoing gastrectomy alone. Therefore, gastrectomy with concomitant cholecystectomy increased cholelithiasis-free survival from 80 to $100 \%$. Assuming a constant accrual rate and an accrual time of 2 years, a minimum follow-up period of 3 years, an overall survival probability of $50 \%$ at 5 years 
[23], 122 patients (61 in the control group and 61 in the cholecystectomy group) are necessary to ensure an $80 \%$ power with an alpha of $5 \%$, when using a two-sided logrank test. Assuming a $50 \%$ drop-out rate during a 3 year follow-up was deemed sufficient to account for both cancer mortality and follow-up loss, expected to be minimal or even null, according to previous IRGGC studies [23, 25].

The Study began the enrollment at the end of 2008 . Patients were randomized into two groups according to a computer generated list: in the first group the patient were submitted to prophylactic cholecystectomy (PC) during standard surgery for curable gastric cancer (subtotal or total gastrectomy), while in the second group they were submitted to standard gastric surgery only (SS). The standard surgery for gastric cancer includes both total and subtotal gastrectomy with lymphadenectomy (D1, D2, D3). The way of surgical approach, either open or laparoscopic, was not relevant for the aim of the study. All types of reconstructions were allowed, including either mechanic or hand-sewn anastomosis. Drainage tubes were left on routine basis, and withdrawn according to surgeon's preference. Cholecystectomy was performed whenever more appropriate for the operating surgeon during gastric cancer surgery and using whichever surgical device and instrumentation preferred by the surgeon. It could have been done either laparoscopically or open, depending on the surgical procedure chosen for the gastrectomy.

The protocol required a complete preoperative data collection, including patient demographics and comorbidities, details of gastric surgery, perioperative treatment and complications and follow-up.

Patient demographics, BMI and perioperative records were electronically sent from each Center participating to the study using a standard pre-settled database (Microsoft Access, Microsoft Corporation Italia, Segrate, Milan, Italy). The study was expected to enroll patients approximately after 1-3 years, according to the pre-assessed mean number of gastrectomies managed monthly in each Centers. The Local Ethic Committee (LEC) of the Leading Center required an interim analysis targeted on safety of prophylactic cholecystectomy during gastric surgery for cancer. This analysis was published in an abstract form [26] when half of the patients (63) cohort was included.

\section{Statistics}

Data were converted from original databases to a more specific electronic statistic platform and matched in the two groups using the SPSS for Windows 18.0 software package (SPSS Inc, Chicago, IL, USA). Significance of differences was evaluated by the non parametric Mann-Whitney $U$ test (and by the Fischer's exact test for categorical variables. A $P$ value $<0.05$ was considered statistically significant.

\section{Results}

After 40 months from the beginning of study, 172 patients, from the 9 participating centers, were eligible for the study. According to the protocol they had a preoperative biopsy of gastric adenocarcinoma, were $\leq 80$ years old, did not have detectable or suspected cholelithiasis, and previous gastric or biliary surgery. They were not affected by hemolytic anemia or genetic hypercholesterolemia and were considered suitable for curative surgery. Ten of them refused to sign the informed consent and, hence, they were not processed into the trial. Therefore 162 patients were randomized. Details of numbers of randomized patients from different Centers are shown in Fig. 1.

These diverse figures are partly due to the time period of participation to the study, which was different for each Center, and to the different number of participating surgeons from the various Centers.

Thirty-two patients were afterwards excluded from the study due to flawing of inclusion criteria (30 not R0 surgery for carcinosis, metastases or positive margins at pathology, 2 definitive pathology reports of lymphoma). The number of patients enrolled and included along the time of recruitment are represented in Fig. 2.

As shown, recruitment rate was quite steady throughout all the time period of the study and proportion between randomized and included patients was similarly stable. This demonstrates that flaws of inclusion criteria were unvarying along the time and among all the participating Centers, being represented mostly by non-curative surgery, which is unfortunately a significant percentage of gastric cancer patients even after a thorough preoperative clinical staging.

For the present end of recruitment analysis, we proceeded to process data of the remaining 130 patients. Figure 3 shows details of the study enrollment at the end of recruitment.

Final analysis included 65 patients who received prophylactic cholecystectomy (PC) and 65 who did not (SS).

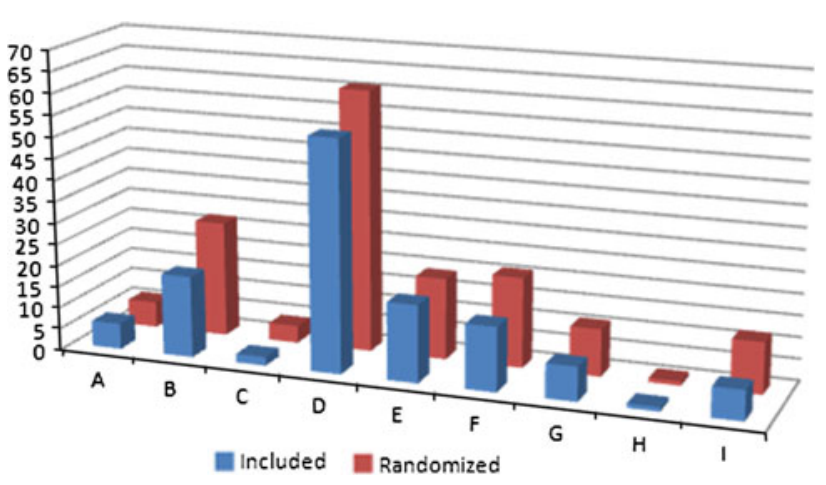

Fig. 1 Patients recruitment according to the participating Centers $(a-i)$ 


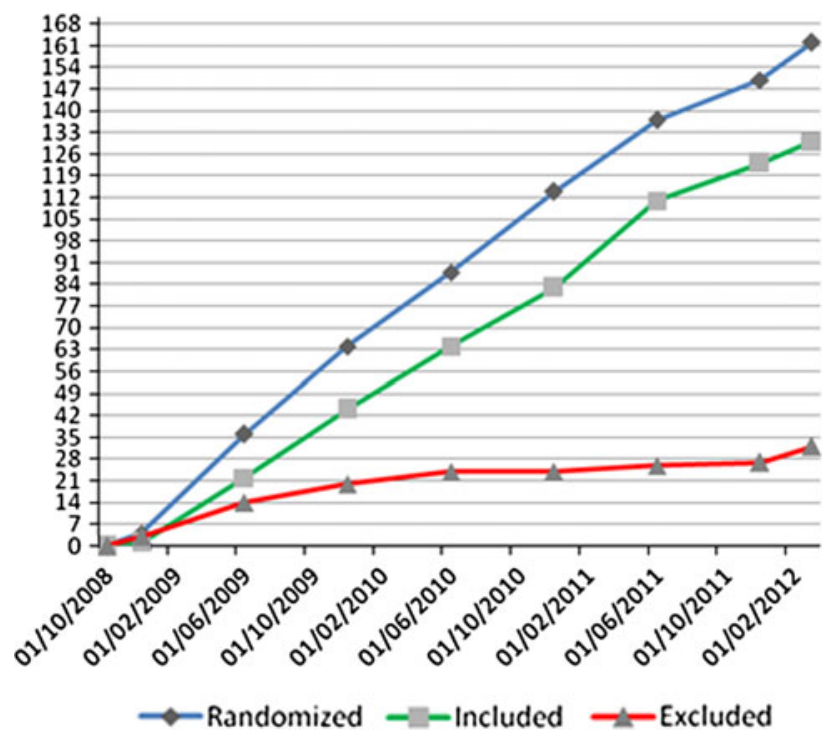

Fig. 2 Patients recruitment throughout the time

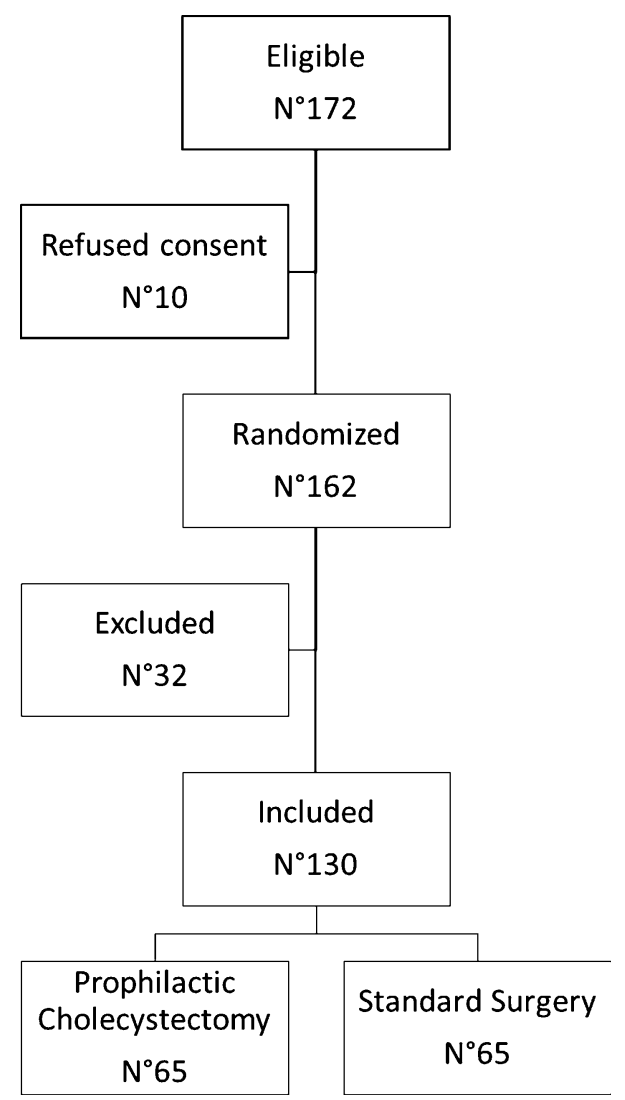

Fig. 3 The Cholegas Study at March 2012 (end of recruitment)

Table 1 summarizes demographics, BMI, operative data and definitive pathological staging of the analyzed patients. None except ALP level showed significant differences between the two groups.
Table 1 Comparison between PC (prophylactic cholecystectomy) and SS (standard gastric surgery only) groups

\begin{tabular}{|c|c|c|c|}
\hline & $\mathrm{PC}(n=65)$ & $\mathrm{SS}(n=65)$ & $P$ value \\
\hline Age (years) & $67(24-80)$ & $69(43-80)$ & 0.89 \\
\hline $\operatorname{Sex}(F / M)$ & $27 / 38$ & $28 / 37$ & 1.00 \\
\hline BMI $\left(\mathrm{kg} / \mathrm{m}^{2}\right)$ & $24(19-37)$ & $24(18-37)$ & 0.28 \\
\hline Bilirubin tot. (mg/dL) & $0.6(0.26-2.34)$ & $0.66(0.28-2.3)$ & 0.91 \\
\hline Bilirubin dir. (mg/dL) & $0.15(0.02-0.4)$ & $0.14(0.01-0.37)$ & 0.82 \\
\hline GGT & $23(4-100)$ & $22(7-51)$ & 0.50 \\
\hline ALP & $100(40-393)$ & $81(35-227)$ & 0.001 \\
\hline \multicolumn{4}{|l|}{ Type of gastrectomy } \\
\hline Subtotal & $46(71)$ & $41(63)$ & 0.456 \\
\hline Total & $19(29)$ & $24(37)$ & \\
\hline \multicolumn{4}{|l|}{ Lymphadenectomy } \\
\hline D1 & $5(8)$ & $7(11)$ & 0.863 \\
\hline D2 & $52(80)$ & $50(77)$ & \\
\hline D3 & $8(12)$ & $8(12)$ & \\
\hline Station 12 & $38(58)$ & $34(52)$ & 0.597 \\
\hline \multicolumn{4}{|l|}{ Reconstruction } \\
\hline Roux & $45(69)$ & $50(77)$ & 0.429 \\
\hline Billroth 2 & $20(31)$ & $15(23)$ & \\
\hline \multicolumn{4}{|l|}{ Tumour stage (pTNM) } \\
\hline Stage IA, B & $25(38)$ & $25(38)$ & 0.748 \\
\hline Stage IIA, B & $6(10)$ & $7(11)$ & \\
\hline Stage IIIA, B, C & $32(49)$ & $33(51)$ & \\
\hline Missing data & $2(3)$ & $0(0)$ & \\
\hline
\end{tabular}

Patient characteristics, details of intervention and staging. Continuous variables are expressed as median and range, categorical data as number and percentage

$B M I$ body mass index, $p T N M$ pathological tumour node metastasis (7th ed)

Table 2 Comparison between PC and SS

\begin{tabular}{lccc}
\hline & PC $(n=65)$ & SS $(n=65)$ & $P$ value \\
\hline $\begin{array}{l}\text { Duration of surgery } \\
\quad(m i n)\end{array}$ & $210(140-350)$ & $210(120-345)$ & 0.25 \\
Blood loss (ml) & $200(50-1000)$ & $200(100-900)$ & 0.82 \\
Duration of TPN (days) & $3(0-16)$ & $3(0-30)$ & 0.69 \\
Duration of TEN (days) & $0(0-15)$ & $0(0-22)$ & 0.94 \\
Biliary complications & $1(1.5)$ & $0(0)$ & 1.00 \\
Surgical complications & $12(18)$ & $6(9)$ & 0.203 \\
Other non-surgical & $9(14)$ & $7(11)$ & 0.790 \\
$\quad$ complications & $16(25)$ & $11(17)$ & 0.387 \\
Overall complications & $1(1.5)$ & $0(0)$ & 1.00 \\
Mortality & $10(7-30)$ & $10(7-45)$ & 0.93 \\
Hospital stay (days) &
\end{tabular}

Perioperative reports and complications: continuous variables are expressed as median and range, categorical data as number and percentage

$T P N$ total parenteral nutrition, $T E N$ total enteral nutrition 
A summary of perioperative results and complications occurred in both groups are shown in Table 2. None of these was significantly different.

Among the PC group, 12 patients had surgical complications, with one of them experiencing more than one $(2$ duodenal fistulas, 3 hemorrhages, 1 anastomotic leak, 1 biliary leak, 2 bowel obstructions, 1 abdominal abscess, 1 wound dehiscence and 2 other complications). Only 1 biliary complications was recorded in PC group. It was a biliary fluid from drainage, which disappeared spontaneously, in few days, without any treatment. No further investigation was carried out to demonstrate if it was coming from the gallbladder site or from a small duodenal dehiscence. Anyway, such a leakage might have been caused by prophylactic cholecystectomy. No other biliary complications, such as biliary ducts injuries, were recorded in the treatment Group. Six patients had surgical complications in the SS group, more than one in two of them (2 hemorrhages, 2 anastomotic leaks, 1 pancreatic stump leakage, 1 bowel perforation, 1 bowel obstruction and 1 other complication).

Among non-surgical complications we registered 9 cases of pleuritis, 2 cases of ARDS, 6 cases of pneumonia, 1 case of pulmonary embolism (which resulted fatal), 2 cases of urinary tract infection and 1 case of acute myocardial infarction. Nine patients had the aforementioned complications in the PC group, while 7 had the same in the SS group. In some cases, in both groups, patients experienced more than a single complication. As mentioned, only one postoperative death occurred, this happened in the PC group due to pulmonary embolism. Differences were not statistically significant.

Other criteria were compared between the two groups, in order to find differences in costs and anesthesiology risks, such as: duration of surgery, blood loss, days of parenteral or enteral nutrition and hospital stay. Once again no differences reached a statistical significance.

\section{Discussion}

Gallstones and gallbladder sludge are known complications after gastrectomy. Many studies reported up to 15-25\% incidence 5 years after gastric surgery [1-6] with a mean value estimated of $17 \%$ in all studies [1-7]. Without a specific evidence in the literature, some authors $[4,11]$ recommend to perform a prophylactic cholecystectomy at the time of gastric surgery, while others do not $[1,7]$ and the decision of gallbladder management is left, to date, to each surgeon's preference.

We designed a trial to find differences in the number of patients who developed symptoms and needed further surgery for cholelithiasis after standard gastric cancer surgery and to find differences in cholelithiasis incidence itself. Of note, the sample size had been planned to detect differences in the incidence of cholelithiasis rather than in the incidence of complications.

After little more than 3 years from the beginning of study, 162 patients were randomized from 9 Centers. 32 (20\%) were then excluded due to flawing of inclusion criteria. Most of the excluded patients had occult distant metastasis, carcinosis or non-curative surgery (R1/R2), despite preoperative assessment of operability achieved by CT-scan of the abdomen. This fact could explain the high drop-off percentage and the relatively longer than expected recruitment period. The mean number of included patients was approximately 22 for each semester throughout the whole study period.

Although every participating Center had a previously certified activity in gastric surgery (members of the IRGGC-Italian Research Group for Gastric Cancer), the number of included patients for each Center was approximately only 5 yearly (mean), but inclusion criteria restricted the candidates for gastric surgery to younger than 80 years, without previous biliary surgery or concomitant cholelithiasis. Furthermore we should consider, as previously stated, that not all Centers entered the study at the same time, with some of them joining the trial after 1 year and others even after 2 years. Besides that, not all the surgeons of every Center agreed to enter the trial.

Demographic and perioperative results were comparable to those reported in previous papers regarding Italian activity of gastric surgery $[23,25,27,28]$ and even to those of some international series [29-33]. Moreover, the two arms of the study were highly comparable regarding patients' characteristics, operative details, results and complications, except for a significant difference in ALP level (higher in PC group), which is not aligned with bilirubin and GGT and cannot be explained by our data. Regarding surgical reconstruction, only Roux-en-Y and Billroth II were recorded. A Billroth I reconstruction might change cholelithiasis pathogenesis and results but it is very rare and not routinely performed in Italy.

Eventually, the aim of this analysis, at the end of the recruitment period, was to rule out an increase of perioperative complications rate due to the prophylactic cholecystectomy, and this was achieved. Moreover, prophylactic cholecystectomy was not burdened by extra time (and costs), i.e. by an elongation of the length of surgery or postoperative stay. This can be explained by the fact that only lymphadenectomy is time consuming and it is equivalent whether or not cholecystectomy is performed. Only one biliary leakage could be attributed to prophylactic cholecystectomy, although not demonstrated by investigators, because it disappeared spontaneously in few days. No major injuries to bile duct were, instead, registered in PC Group. 
Among the first group, 12 patients had surgical complications, with one of them experiencing more than one ( 2 duodenal fistulas, 3 hemorrhages, 1 anastomotic leak, 1 biliary leak, 2 bowel obstructions, 1 abdominal abscess, 1 wound dehiscence and 2 other complications), only 1 biliary leakage might have been caused by prophylactic cholecystectomy. While 6 patients had surgical complications in the second group, sometimes more than one ( 2 hemorrhages, 2 anastomotic leaks, 1 pancreatic stump leakage, 1 bowel perforation, 1 bowel obstruction and 1 other complication).

Even though surgical complications were exactly twice as common, in PC Group of those found in SS, such complications were not due to prophylactic cholecystectomy, except, maybe, in one case, as already explained, and such a difference didn't reach a statistical significance, even though the sample size was not dimensioned to find such differences. One postoperative death occurred in the treatment group due to pulmonary embolism.

Non-surgical complications were equally distributed and mainly involving lungs, as commonly found in upper-GI surgery. Similarly no differences were significant in duration of surgery, blood loss and hospital stay; thus demonstrating that prophylactic cholecystectomy is a quite easy procedure, when performed during another surgical operation and doesn't change the anesthesiology load to the patient and hospital costs at all.

In this end of recruitment analysis, including 130 patients operated for gastric malignancy, the group of patients who received prophylactic cholecystectomy did not experience any additional perioperative complications related to biliary surgery. Moreover, no additional time and costs were added to the standard gastric cancer surgical procedure, due to comparable duration of surgery and length of postoperative stay. These results show us that prophylactic cholecystectomy, during gastric cancer surgery, is safe and not time and cost consuming. Nonetheless, whether it might be considered effective and useful for the patients, in order to prevent long-term impairment of quality of life, due to biliary complications and a second intervention, has yet to be investigated by the results of the present study follow-up.

\section{Conflict of interest None.}

\section{References}

1. Kobayashi T, Hisanaga M, Kanehiro H, et al. Analysis of risks factors for the development of gallstones after gastrectomy. Br J Surg. 2005;92:1399-403.

2. Sanders G, Kingsnorth AN. Gallstones. BMJ. 2007;335:295-9.

3. Sakorafas GH, Milingos D, Peros G. Asymptomatic cholelithiasis: is cholecystectomy really needed? Dig Dis Sci. 2007;52:1313-25.
4. Fukagawa T, Katai H, Saka M, et al. Gallstone formation after gastric cancer surgery. J Gastrointest Surg. 2009;13:886-9.

5. Rehnberg O, Haglung U. Gallstone disease following antrectomy and gastroduodenostomy with or without vagotomy. Ann Surg. 1985;201:315-8.

6. Li VKM, Pulido N, Martinez-Suartez P, et al. Symptomatic gallstones after sleeve gastrectomy. Surg Endosc. 2009;23: 2488-92.

7. Gillen S, Michalski CW, Schuster T, et al. Simultaneous/incidental cholecystectomy during gastric/esophageal resection: systematic analysis of risks and benefits. World J Surg. 2010;34:1008-14.

8. Inoue K, Fuchigami A, Higashide S, et al. Gallbladder sludge and stone formation in relation to contractile function after gastrectomy. Ann Surg. 1992;215:19-26.

9. Qvist N. Review article: gall-bladder motility after intestinal surgery. Aliment Pharmacol Ther. 2000;14(s2):35-8.

10. Yi SQ, Ohta T, Tsuchida A, et al. Surgical anatomy of innervation of the gallbladder in humans and Suncus murinus with special reference to morphological understanding of gallstone formation after gastrectomy. World $\mathrm{J}$ Gastroenterol. 2007;14:2066-71.

11. Wu CC, Chen CY, Wu TC, et al. Cholelithiasis and cholecystitis after gastrectomy for gastric carcinoma: a comparison of lymphadenectomy of varying extent. Hepatogastroenterology. 1995;42:867-72.

12. Tomita R, Tanjoh K, Fujisaki S. Total gastrectomy reconstructed by interposition of a jejunal $\mathrm{J}$ pou with preservation of hepatic vagus branch and lower esophageal sphincter for $\mathrm{T} 2$ gastric cancer without lymph node metastasis. Hepatogastroenterology. 2004;51:1233-40.

13. Akatsu T, Yoshida M, Kubota T, et al. Gallstone disease after extended (D2) lymph node dissection for gastric cancer. World J Surg. 2005;29:182-6.

14. Watemberg S, Landau O, Avrahami R, et al. Incidental cholecystectomy in the over-70 age group. A 19-year retrospective, comparative study. Int Surg. 1997;82:102-4.

15. Juhasz EZ, Wolff BG, Meagher AP, et al. Incidental cholecystectomy during colorectal surgery. Ann Surg. 1994;219:467-74.

16. Wolff BG. Current status of incidental surgery. Dis Colon Rect. 1995;38:435-41.

17. Sasaki A, Nakajima J, Nitta H, et al. Laparoscopic cholecystectomy in patients with a history of gastrectomy. Surg Today. 2008;38:790-4.

18. Fraser SA, Sigman H. Conversion in laparoscopic cholecystectomy after gastric resection: a 15-year review. Can J Surg. 2009;52:463-6.

19. Oh SJ, Choi WB, Song J, et al. Complications requiring reoperation after gastrectomy for gastric cancer: 17 years experience in a single institute. J Gastrointest Surg. 2009;13:239-45.

20. Liu XS, Zhang Q, Zhong J, et al. Acute cholecystitis immediately after radical gastrectomy: a report of three cases. World J Gastroenterol. 2010;16:2702-4.

21. Kwon AH, Inui H, Imamura A, et al. Laparoscopic cholecystectomy and choledocholithotomy in patients with a previous gastrectomy. J Am CollSurg. 2001;193:614-9.

22. Lepage C, Sant M, Verdecchia A, et al. Operative mortality after gastric cancer resection and long-term survival differences across Europe. Br J Surg. 2010;97:235-9.

23. Marrelli D, Pedrazzani C, Morgagni P, on behalf of the Italian Research Group for Gastric Cancer (IRGGC), et al. Changing clinical and pathological features of gastric cancer over time. Br J Surg. 2011;98:1273-83.

24. Farsi M, Bernini M, Bencini L, GIRCG (Gruppo Italiano di Ricerca sul Cancro Gastrico), et al. The CHOLEGAS study: multicentric randomized, blinded, controlled trial of gastrectomy 
plus prophylactic cholecystectomy versus gastrectomy only, in adults submitted to gastric cancer surgery with curative intent. Trials. 2009;15:10-32.

25. Verlato G, Roviello F, Marchet A, et al. Indexes of surgical quality in gastric cancer surgery: experience of an Italian network. Ann SurgOncol. 2009;16:594-602.

26. Bernini M, Doglietto GB, Berardi S, et al. IRGGC. The Cholegas study: safety of prophylactic cholecystectomy during gastrectomy for cancer: an interim analysis. Abstract Book 10th IGCC Seoul 2011:FP 9-8.

27. Baiocchi GL, Tiberio GA, Minicozzi AM, et al. A multicentric Western analysis of prognostic factors in advanced, node-negative gastric cancer patients. Ann Surg. 2010;252:70-3.

28. Degiuli M, Sasako M, Italian Gastric Cancer Study Group. Morbidity and mortality in the Italian Gastric Cancer Study Group randomized clinical trial of D1 versus D2 resection Shi Y,
Zhou Y. The role of surgery in the treatment of gastric cancer. Br J Surg. 2010;97:643-9.

29. Shi Y, Zhou Y. The role of surgery in the treatment of gastric cancer. J Surg Oncol. 2010;101:687-92.

30. Kodera E, Fujiwara M, Ito Y, et al. Radical surgery for gastric carcinoma: it is not an issue of whether to perform D1 or D2. Dissect as many lymph nodes as possible and you will be rewarded. Acta Chir Belg. 2009;109:27-35.

31. Saka M, Morita S, Fukagawa T, et al. Present and future status of gastric cancer surgery. Jpn J Clin Oncol. 2011;41:307-13.

32. Hartgrink HH, Jansen EP, van Grieken NC, et al. Gastric cancer. Lancet. 2009;374:477-90.

33. Enzinger PC, Benedetti JK, Meyerhardt JA, et al. Impact of hospital volume on recurrence and survival after surgery for gastric cancer. Ann Surg. 2007;245:426-34. 